N. MANIKANDAN

B.H. SHARMILA

S. ASOKAN

\section{Photoconductivity studies on bulk As-Te-In glasses}

Department of Instrumentation, Indian Institute of Science, Bangalore 560012, India

\begin{abstract}
Photocurrent measurements have been undertaken on bulk As40 $\mathrm{Te}_{60-x} \operatorname{In}_{x}$ glasses of different compositions. It has been found that the photocurrent increases with illumination, which can probably be understood on the basis of the large dielectric constant of these materials and also due to the presence of a larger number of positive defect states. Further, the composition dependence of the conductivity activation energy and the photosensitivity exhibit a maximum at $x=12.5(\langle r\rangle=2.65)$ and a minimum at $x=15(\langle r\rangle=2.70)$. Earlier investigations on As-Te-In glasses, such as high-pressure resistivity behavior, electrical switching, etc., have identified the compositions $x=12.5$ and $x=15$ as the rigidity percolation threshold (RPT) and the chemical threshold (CT) respectively. Based on this, the maximum and the minimum seen in activation energy and photosensitivity of $\mathrm{As}_{40} \mathrm{Te}_{60-x} \mathrm{In}_{x}$ glasses at $x=12.5$ and $x=15$ can be attributed to RPT and CT respectively.
\end{abstract}

PACS 72.40.w; 71.55.Jv; 72.80.Ng

\section{Introduction}

Investigations on the electrical transport properties of amorphous semiconductors have been stimulated by their potential technological applications. Photocurrent measurements on chalcogenide glasses are particularly important for applications of these materials in xerography and photovoltaic. Further, photocurrent measurements yield information about the defect states present in the material, which in turn provide an idea about the recombination processes [1]. Compared to amorphous thin films, only few studies have been reported in literature on the photoconductivity of bulk glassy samples $[2,3]$. In most of the reported studies, a decrease in photocurrent (photo degradation) has been found to occur under illumination, which has been attributed to the formation of light induced metastable defects (LIMDs) in the system [4].

In the present study, photocurrent measurements have been undertaken on bulk As-Te-In glasses. The photocurrent has been found to increase with illumination, in contrast to

Fax: +91-80-3600135, E-mail: sasokan@isu.iisc.ernet.in the earlier observations [2]. The anomalous variation in photocurrent has been understood in terms of the bonding of metal atoms in chalcogenides and its influence on the defects present in the system. The composition dependence of photosensitivity and the activation energy under dark \& light illuminated states has been found to provide an idea about the topological thresholds present in the system.

\section{2}

\section{Experimental details}

Bulk $\mathrm{As}_{40} \mathrm{Te}_{60-x} \operatorname{In}_{x}(7.5 \leq x \leq 16.5)$ samples were prepared by melt quenching technique. Arsenic, tellurium and indium (99.999\% pure) were weighed appropriately and taken in a quartz ampoule. The ampoules were evacuated to about $10^{-5}$ Torr and sealed. The sealed ampoules were loaded in a furnace and the temperature of the furnace was increased at a rate of $100^{\circ} \mathrm{C} / \mathrm{hr}$ up to $950^{\circ} \mathrm{C}$. The ampoules were maintained at this temperature for about 24 hours and then quenched in ice water- $\mathrm{NaOH}$ mixture. The amorphous nature of the samples was confirmed by X-ray diffraction experiments.

Samples were cut to a size of around $0.8 \mathrm{~mm}$ thickness and loaded in the photoconductivity cell, consisting of a stainless steel body and a top flange with a liquid nitrogen trap. The two flat bottom brass electrodes were attached to the trap through a copper block. Samples were placed between the electrodes under an electric field of $150 \mathrm{~V} / \mathrm{cm}$ and the whole cell was evacuated to $10^{-3}$ Torr. A $150 \mathrm{~W}$ Xe lamp (Oriel Corporation model no 6136) and $514 \mathrm{~nm}$ Ar-ion laser were used as the source of light. Intensity on the sample was maintained at $125 \mathrm{~mW}$. Illumination was done using an optical fiber through a quartz window. This ensures that there is no loss of intensity either by scattering or absorption.

The variation of current with temperature (123 K to $293 \mathrm{~K}$ ) was measured to determine the activation energy of the sample. The growth and decay of photocurrent was observed, by illuminating and then cutting off the light in a time interval of 10 min each.

\section{$3 \quad$ Results}

Figure 1 shows the variation of dark and photo current of $\mathrm{As}_{40} \mathrm{Te}_{60-x} \mathrm{In}_{x}$ glasses with temperature. It can be seen 


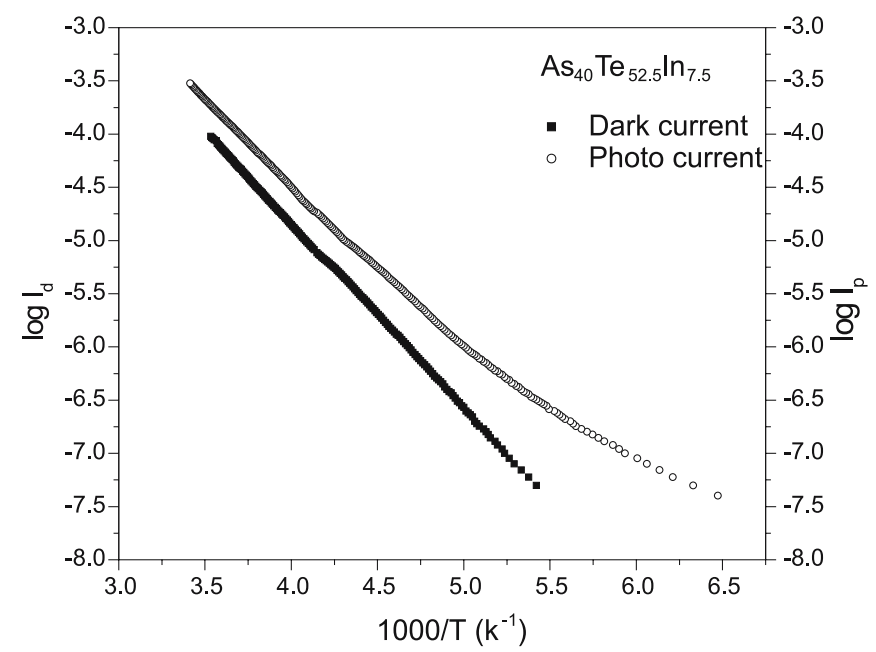

FIGURE 1 Temperature dependence of dark and photocurrent of As-Te-In glasses

that the dark current increases linearly with temperature in the range $123 \mathrm{~K}$ to $293 \mathrm{~K}$, whereas the photocurrent shows a nonlinear dependence in the lower temperature ranges and a linear variation in the range of $243 \mathrm{~K}$ to $293 \mathrm{~K}$. The activation energy $(\Delta E)$ of the samples, under dark conditions, has been found to lie in the range of 0.34 to $0.40 \mathrm{eV}$, depending on the composition $(x)$. The conductivity activation energy under illumination, obtained from the linear region, has been found to lie in the range 0.33 to $0.37 \mathrm{eV}$. It is also found that the temperature dependence of photocurrent does not show any maximum in the observed range. Figure $2 \mathrm{a}$ and $\mathrm{b}$ shows the dependence on composition/average coordination number $(<r>)$, of the conductivity activation energy under dark and light illuminated states. For calculating $\langle r\rangle$, the coordination numbers of arsenic and tellurium have been taken to be 3 and 2 respectively. Further, a coordination of four has been assumed for indium on the basis of Kastner's proposition that metal impurities in chalcogenide glasses coordinate tetrahedrally [5]. Experimental investigations also support tetrahedral coordination of metal atoms in glassy tellurides [6,7], whereas they are mostly three fold coordinated in selenide glasses [8-10]. However, there is a possibility of multiple coordinations of indium atoms (four and six) in As-Te-In glasses, as in the case of aluminum in Al-As-Te samples [11], which has not been experimentally explored. Hence, the average coordination numbers indicated can only be taken as suggestive and not exact.

Figure 2 shows that the activation energy under dark and light illuminated states exhibit a maximum at $x=$ $12.5(<\mathrm{r}>=2.65)$ and a minimum at $x=15(<r>=2.70)$. The investigations on the electrical switching \& high-pressure resistivity behavior $[12,13]$ and thermal studies [14] on $\mathrm{As}_{40} \mathrm{Te}_{60-x} \mathrm{In}_{x}$ glasses have also shown specific signatures at these compositions. These studies have identified the composition $x=12.5(<r>=2.65)$ as the Rigidity Percolation Threshold and $x=15(<r>=2.70)$ as the Chemical Threshold of the system. Based on this, we can associate the maximum and minimum seen in the conductivity activation energy under dark and light illuminated conditions at
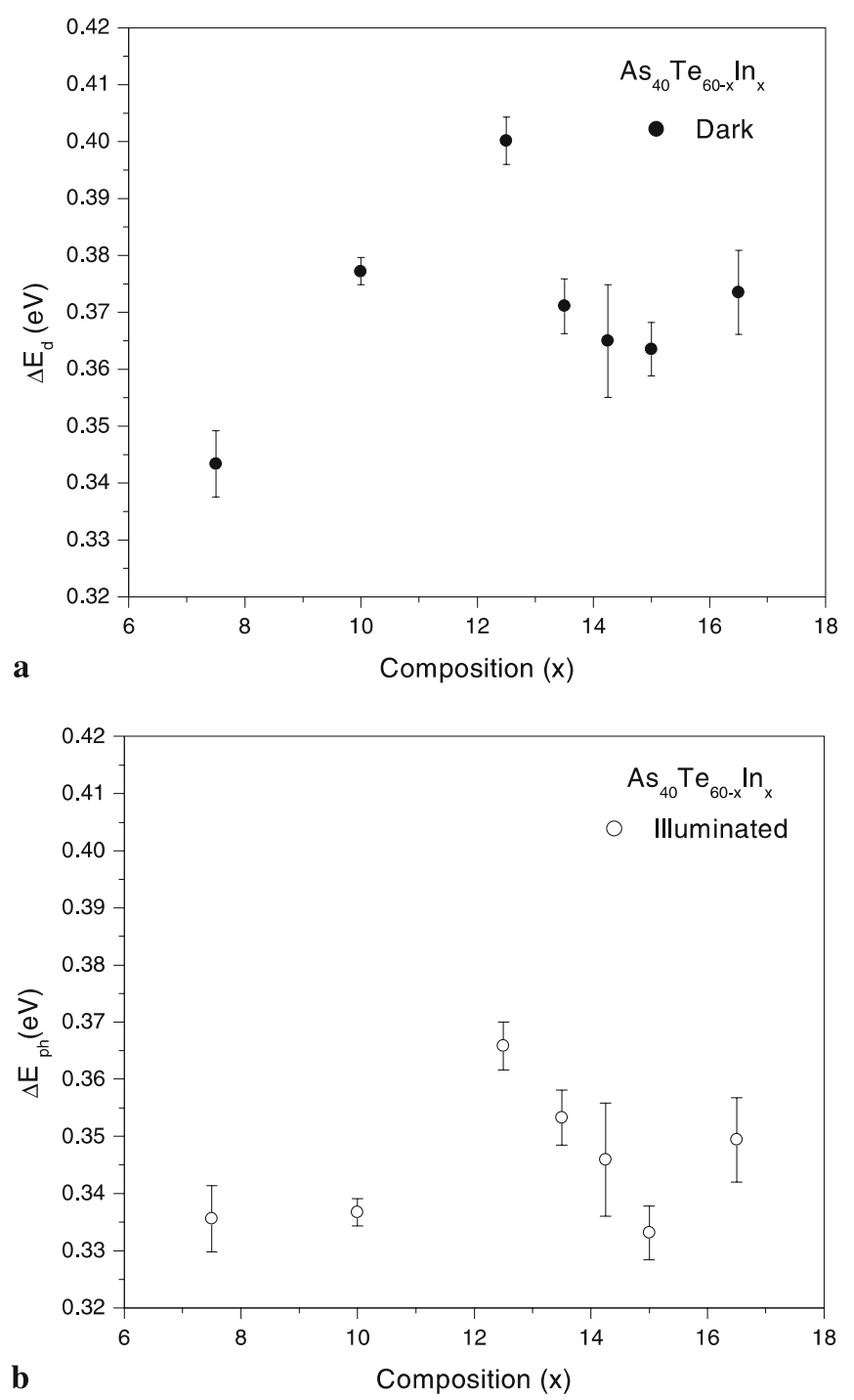

FIGURE 2 Variation of activation energy of As-Te-In glasses with composition

$x=12.5(<r>=2.65)$ and at $x=15(<r>=2.70)$ with the rigidity percolation and chemical thresholds, respectively.

The time dependence of photocurrent is shown in Fig. 3, which indicates that the photocurrent increases continuously when the sample is exposed to light and a decrease in current is seen when the light is put off.

The variation of photosensitivity, which is the ratio between the photocurrent and the dark current, with composition/average coordination number $\langle r\rangle$, is shown in Fig. 4. It is observed that the photosensitivity also shows specific signatures at $x=12.5$ and $x=15$.

Figure 5 shows the variation in photocurrent with the intensity of the incident illumination, which indicates that the photocurrent exhibits a linear dependence on intensity.

4

4.1

\section{Discussions}

\section{Absence of photo-degradation}

In chalcogenide glasses, the photocurrent is found to decrease with illumination due to photo degradation $[2,4]$. It has been proposed by Shimakawa et al. [4] that the de- 


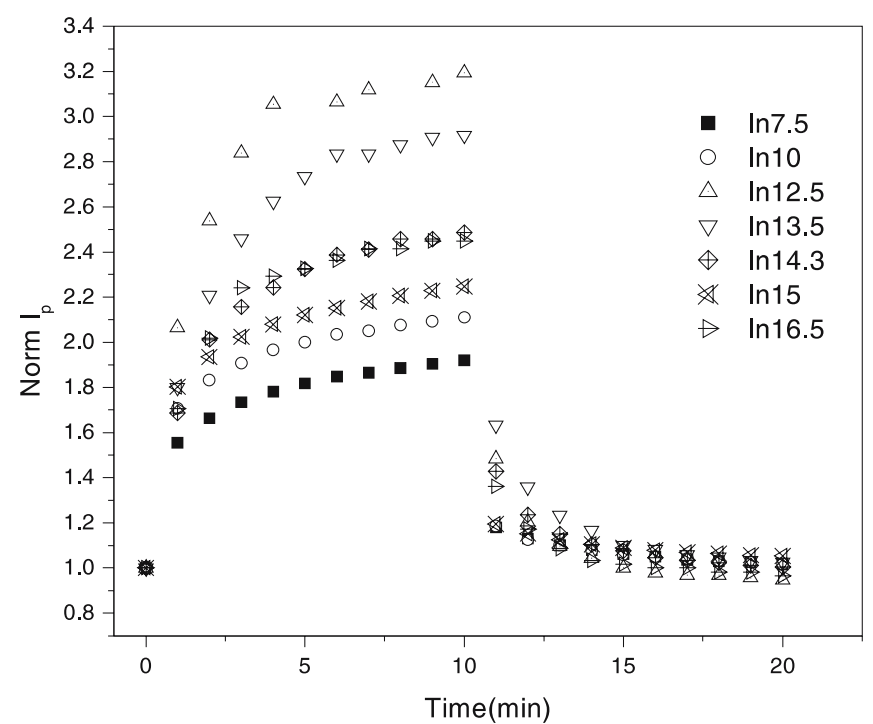

FIGURE 3 Time dependence of photocurrent of As-Te-In glasses

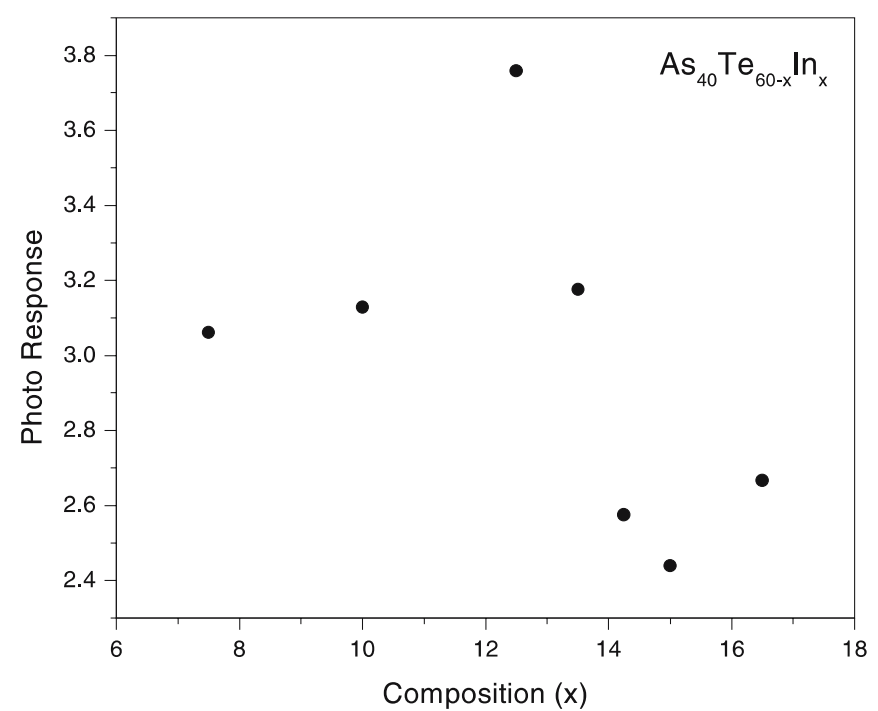

FIGURE 4 Variation of photosensitivity of As-Te-In glasses with composition

crease in photocurrent is because of the formation of light induced metastable defects, which are produced due to the bond conversion between the random pairs and the intimate valence alternation pairs (IVAPs). Further, in materials with smaller band-gap, the large dielectric constant reduces the Coulomb attraction between the charges, which leads to the non-formation of random pairs and intimate pairs [15]. The present study clearly shows that there is no photo degradation in As-Te-In glasses. The previous investigations reveal that the band-gap of $\mathrm{As}_{40} \mathrm{Te}_{60-x} \mathrm{In}_{x}$ glasses is smaller, lying in the range 0.6 to $0.70 \mathrm{eV} \mathrm{[13],} \mathrm{which} \mathrm{is} \mathrm{consistent} \mathrm{with} \mathrm{the} \mathrm{absence}$ of photo degradation.

Based on the 8-N rule [16], one can expect indium atoms to be threefold coordinated in a chalcogenide network. Further, metal impurities coordinate tetrahedrally in chalcogenide semiconductors (especially in tellurides), making coordinate bonds with neighboring chalcogen atoms utilizing their lone pairs [5]. The trivalent atoms such as Al, In, Ga etc are expected to have one coordinately bonded and three cova-

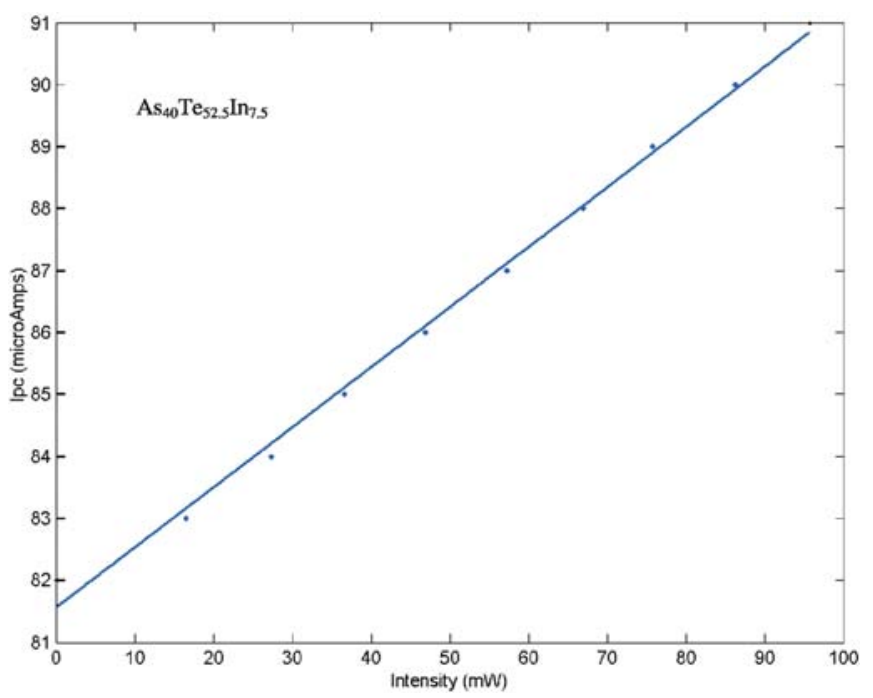

FIGURE 5 Intensity dependence of photocurrent of As-Te-In glasses

lently bonded chalcogen neighbors. The coordinate bonding leads to the formation of $\mathrm{In}_{4}^{-}$and $\mathrm{Te}^{+}\left(\mathrm{C}_{3}^{+}\right)$centers that are linked together by donor acceptor bonding and Coulomb attraction [13]. Thus, the tetrahedral coordination of indium results in an increase in $\mathrm{C}_{3}^{+}$centers. As the photo-degradation has been found to be mainly due to hole trapping by $\mathrm{C}_{1}^{-}$centers, the increase in $\mathrm{C}_{3}^{+}$centers may also play a role in the reduction in photo-degradation.

Upon prolonged illumination, the additional positively charged light excited electrons, leading to formation of $\mathrm{C}_{3}^{0}$ centers, passivate defect centers. The $\mathrm{C}_{3}^{0}$ defects formed as above may subsequently decay to $C_{1}^{0}$ centers [17]. When the illumination is put off, the excess $C_{1}^{0}$ centers formed will transform into $\mathrm{C}_{3}^{+}$and $\mathrm{C}_{1}^{-}$centers, leading to a drop in the photocurrent [18], which is found to be exponential.

\subsection{Intensity dependence of photocurrent}

The variation of photocurrent $(F)$ with intensity $(I)$ of incident light is found to obey a relationship,

$F \propto I^{\gamma}$.

The value of $\gamma$ determines the type of recombination mechanism occurring in the system. The excess electrons and holes can either recombine among themselves or recombine with the charged centers. The former leads to a bimolecular recombination (BMR) and the latter to monomolecular recombination (MMR) [19]. The value of $\gamma$ for BMR and MMR are 0.5 and 1.0, respectively. In the system studied, it is found that $\gamma$ varies from 0.38 to 1.0 for different compositions indicating the presence of a continuous distribution of localized or trap states in the gap of the material [20].

\subsection{Composition dependence}

The variation of activation energy of As-Te-In glasses in the dark and the illuminated states as well as the photosensitivity show a similar trend with composition. Both the activation energy and the photosensitivity have been found to increase with composition initially and attain a maximum 
at $x=12.5(<r>=2.65)$. Thereafter they follow a decreasing trend until they reach a minimum at $x=15(<r>=2.7)$. The addition of more metallic impurities, in general, is expected to lower the activation energy. However, the increase in activation energy with composition/average co-ordination number $\langle r\rangle$ shows that rigidity effects are more dominant than the metallicity factor. As mentioned earlier, the maximum seen in the above properties at $x=12.5$ is associated with the RPT and the minimum is connected with the CT.

\section{$5 \quad$ Conclusions}

Photocurrent measurements on $\mathrm{As}_{40} \mathrm{Te}_{60-x} \mathrm{In}_{x}$ $(7.5 \leq x \leq 16.5)$ glasses show an increase in current with illumination, which is a feature observed in smaller bandgap materials. The absence of photo-degradation may also be due to an increase in the number of $\mathrm{C}_{3}^{+}$centers compared to $\mathrm{C}_{1}^{-}$defects. The variations in conductivity activation energy under dark \& illuminated conditions and the photosensitivity with composition, have been found to show specific features at compositions $x=12.5(\langle r\rangle=2.65)$ and $x=15(\langle r\rangle=2.70)$, which have been identified earlier as the rigidity percolation and chemical thresholds, respectively.

\section{REFERENCES}

1 A.E. Owen, A.P. Firth, P.J.S. Ewen: Phil. Mag. B 52, 347 (1985)

2 N. Toyosowa, K. Tanaka: Phys. Rev. B 56, 7416 (1997)

3 S. Murugavel, S. Asokan: J. Non-Cryst. Solids 303, 296 (2002)

4 K. Shimakawa, S. Inami, T. Kato, S.R. Ellott: Phys. Rev. B 46, 10062 (1992)

5 M. Kastner: Phil. Mag. B 37, 127 (1978)

6 A. Fehrat, R. Olltrault-Fichet, V. Mastelaro, S. Benazeth, R.J. Rivert: J. Phys. (Paris) C2, 201 (1992)

7 K. Ramesh, S. Asokan, K.S. Sangunni, E.S.R. Gopal: J. Phys. Condens. Matter 11, 3897 (1999)

8 R.H. Dejus, S. Susman, K.J. Volin, D.G. Mantague, D.L. Price: J. NonCryst. Solids 143, 162 (1992)

9 C.J. Benmore, P.S. Salmon: J. Non-Cryst. Solids 156-158, 720 (1993)

10 G. Saffarani: Phys. Status Solidi B 213, 261 (1993)

11 S. Murugavel, S. Asokan: Phys. Rev. B 58, 3022 (1998)

12 J.T. Devaraju, B.H. Sharmila, S. Asokan, K.V. Acharya: Phil. Mag. B 81 $583(2001)$

13 B.H. Sharmila, J.T. Devaraju, S. Asokan: J. Non-Cryst. Solids 303, 372 (2002)

14 B.H. Sharmila, J.T. Devaraju, S. Asokan: J. Non-Cryst. Solids 326-327, 154 (2003)

15 K. Hayashi, Y. Hikida, K. Shimakawa, S.R. Elliott: Phil. Mag. Lett 76, 233 (1997)

16 N.F. Mott: Phil. Mag. 19, 832 (1969)

17 D. Vanderbilt, J.D. Joannopoulos: Phys. Rev. B 22, 2927 (1980)

18 F.T. Reis, I. Chambouleyron: J. Non-Cryst. Solids 299-302, 179 (2002)

19 N. Qamhieh, G.J. Adriaenssens: J. Non-Cryst. Solids 292, 80 (2001)

20 A. Rose: Concepts in photoconductivity (Interscience, New York 1963) 